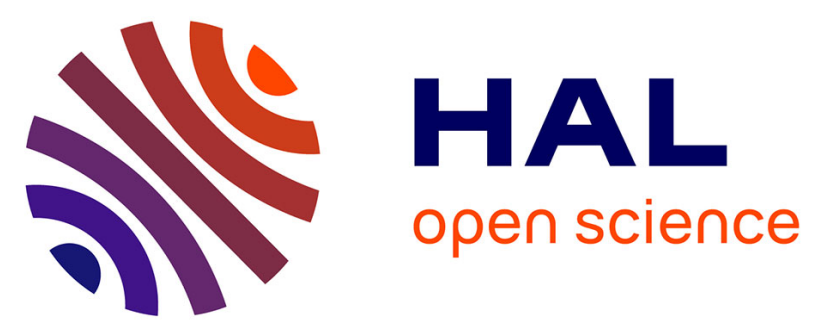

\title{
Statistical Relationship Between Interplanetary Magnetic Field Conditions and the Helicity Sign of Flux Transfer Event Flux Ropes
}

R. Kieokaew, B. Lavraud, N. Fargette, A. Marchaudon, V. Génot, C. Jacquey, D. Gershman, B. Giles, R. Torbert, J. Burch

\section{To cite this version:}

R. Kieokaew, B. Lavraud, N. Fargette, A. Marchaudon, V. Génot, et al.. Statistical Relationship Between Interplanetary Magnetic Field Conditions and the Helicity Sign of Flux Transfer Event Flux Ropes. Geophysical Research Letters, 2021, 48 (6), pp.e2020GL091257. 10.1029/2020GL091257. hal-03188526

\section{HAL Id: hal-03188526 \\ https://hal.science/hal-03188526}

Submitted on 2 Apr 2021

HAL is a multi-disciplinary open access archive for the deposit and dissemination of scientific research documents, whether they are published or not. The documents may come from teaching and research institutions in France or abroad, or from public or private research centers.
L'archive ouverte pluridisciplinaire HAL, est destinée au dépôt et à la diffusion de documents scientifiques de niveau recherche, publiés ou non, émanant des établissements d'enseignement et de recherche français ou étrangers, des laboratoires publics ou privés. 


\section{Geophysical Research Letters}

\author{
RESEARCH LETTER \\ 10.1029/2020GL091257 \\ Key Points: \\ - The helicity sign of 84 flux transfer \\ events (FTEs) is studied using force- \\ free flux rope model fitting \\ - Right-handed (left-handed) FTE \\ flux ropes are mostly preceded by \\ positive (negative) interplanetary \\ magnetic field (IMF) $B_{Y}$ \\ - This IMF $B_{Y}$ control of the helicity \\ sign is compatible with a multiple \\ $\mathrm{X}$-line formation mechanism
}

Supporting Information:

- Supporting Information S1

Correspondence to:

R. Kieokaew,

rkieokaew@irap.omp.eu

Citation:

Kieokaew, R., Lavraud, B., Fargette, N., Marchaudon, A., Génot, V., Jacquey,

C., et al. (2021). Statistical relationship between interplanetary magnetic field conditions and the helicity sign of flux transfer event flux ropes. Geophysical Research Letters, 48, e2020GL091257. https://doi.org/10.1029/2020GL091257

Received 13 OCT 2020 Accepted 15 FEB 2021

(c) 2021. American Geophysical Union. All Rights Reserved.

\section{Statistical Relationship Between Interplanetary Magnetic Field Conditions and the Helicity Sign of Flux Transfer Event Flux Ropes}

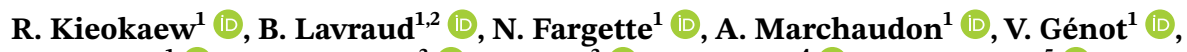

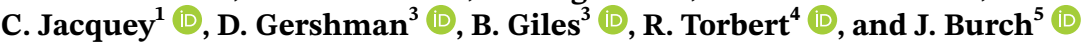 \\ ${ }^{1}$ Institut de Recherche en Astrophysique et Planétologie, CNRS, UPS, CNES, Université de Toulouse, Toulouse, \\ France, ${ }^{2}$ Laboratoire d'Astrophysique de Bordeaux, University of Bordeaux, CNRS, Pessac, France, ${ }^{3}$ NASA Goddard \\ Space Flight Center, Greenbelt, MD, USA, ${ }^{4}$ Space Science Center, University of New Hampshire, Durham, NH, USA, \\ ${ }^{5}$ Southwest Research Institute, San Antonio, TX, USA
}

\begin{abstract}
Flux transfer events (FTEs) are transient phenomena produced by magnetic reconnection at the dayside magnetopause typically under southward interplanetary magnetic field (IMF) conditions. They are usually thought of as magnetic flux ropes with helical structures forming through patchy, unsteady or multiple X-line reconnection. While the IMF often has a non-zero $B_{Y}$ component, its impacts on the FTE flux rope helicity remain unknown. We survey Magnetospheric Multiscale (MMS) observations of FTE flux ropes during years 2015-2017 and investigate the solar wind conditions prior to the events. By fitting a force-free flux rope model, we select 84 events with good fits and obtain the helicity sign (i.e., handedness) of the flux ropes. We find that positive (negative) helicity flux ropes are mainly preceded by positive (negative) $B_{Y}$ component. This finding is compatible with flux ropes formed through a multiple $\mathrm{X}$-lines mechanism.
\end{abstract}

Plain Language Summary The Earth's near-space environment is very dynamic with transient phenomena triggered by interaction between the solar wind and the Earth's magnetopause. The solar wind carries along an interplanetary magnetic field (IMF) whose orientation determines the dynamics of the interaction. When the IMF is southward, magnetic reconnection can be triggered at the Earth's magnetopause on the dayside. A flux transfer event (FTE) is a transient portal that allows the bursty transfer of solar wind into the Earth's magnetosphere. An FTE is envisaged as a twisted magnetic field structure with helical field that looks like a rope. We study the relationship between the IMF orientations and the twist direction of an ensemble of FTEs observed by NASA's Magnetospheric Multiscale mission, by modeling FTEs as magnetic flux ropes. We found that the flux rope twist direction is controlled by the IMF orientation, such that the rope is twisted in the left-handed or right-handed sense depending on the east-west component of the IMF. This result supports the formation of FTEs by a multiple reconnection mechanism.

\section{Introduction}

A flux transfer event (FTE) is a transient phenomenon generated at magnetospheric magnetopauses, and has been most studied at the Earth. It is recognized in spacecraft data as a bipolar magnetic field variation in the direction normal to the magnetopause $\left(B_{N}\right)$, with enhanced core field (C. Russell \& Elphic, 1978). This magnetic field profile suggests a magnetic flux rope structure with helicoidal field. Various formation mechanisms have been proposed for FTEs, such as transient and patchy dayside reconnection (C. Russell \& Elphic, 1978), single X-line with unsteady reconnection rate (Scholer, 1988; D. J. Southwood et al., 1988), and multiple X-line reconnection (Lee \& Fu, 1985; Raeder, 2006). In recent years, there are growing evidence supporting multiple X-line reconnection mechanisms (e.g., Hasegawa et al., 2010; Øieroset et al., 2011). In the Lee \& Fu's model, three reconnection X-lines are assumed to simultaneously exist in the presence of non-zero $B_{Y}$, leading to production of two helical flux tubes (with the same helical sense). In the Raeder's model, FTEs only develop when the dipole tilt is large; they are formed as a result of non-stationary, sequential generation of new X-lines. 
Since solar wind conditions control magnetic reconnection at the Earth's magnetopause, they should control the nature and properties of FTEs. Early spacecraft surveys revealed that FTEs are strongly associated with southward IMF conditions (Berchem \& Russell, 1984; C. Russell et al., 1996) consistent with generation from reconnection at low latitudes (e.g., Paschmann et al., 1982). There is no strong control from other solar wind parameters such as plasma beta, dynamic pressure, and Mach number on the FTE occurrence (Kuo et al., 1995; Wang et al., 2006). The occurrence of FTEs is found to depend on on the IMF orientation but not on its magnitude (Wang et al., 2006). The effect of the IMF $B_{Y}$ component was studied in relation to the spatial distribution and motion of FTEs (e.g., Fear et al., 2012; Karlson et al., 1996). In particular, Karlson et al. (1996) study dayside aurorae as ionospheric signatures of FTEs; they found that there are preferences for auroral events to occur toward dawn for positive $B_{Y}$ and toward dusk for negative $B_{Y}$. The FTE locations are found to correspond to IMF clock angle when taking into account the seasonal bias (e.g., the change in geomagnetic dipole tilt), though there is no clear control of the effective dipole tilt (e.g., combining the dipole tilt with the IMF cone angle) on the location of FTE signatures (Fear et al., 2012). However, direct studies on relationships between the IMF $B_{Y}$ and FTE topologies themselves are limited to event studies (Eastwood et al., 2012).

FTEs are known to have twisted interior field (e.g., Cowley, 1982; Saunders et al., 1984) with a field-aligned core field and an azimuthal field increasing away from the core. Twisting features of FTEs have been theoretically evaluated in terms of magnetic helicity (Song \& Lysak, 1989; Wright \& Berger, 1989, 1990). Magnetic helicity is a measure that can quantify magnetic field topology into twist, shear, linking, and kinking of magnetic fields. It is defined as $H=\int_{V} \mathbf{A} \cdot \mathbf{B} d^{3} r$, where $H$ is the total helicity of the entire magnetic field in a volume $V, \mathbf{B}$ is the magnetic field, $\mathbf{A}$ is the vector potential of $\mathbf{B}$ (i.e., $\mathbf{B}=\nabla \times \mathbf{A}$ ), and $d^{3} \mathbf{r}$ is the differential volume element. Here we use the definition of magnetic helicity to describe the twist of an FTE flux rope and we will only consider its sign. The twist direction around the core field can be characterized as the "handedness" or "sense/sign of the helicity" of the flux rope. The sign of flux rope helicity was studied mostly in magnetic clouds (e.g., Bothmer \& Schwenn, 1998) and in flux ropes at Venus, Mars, and Titan (e.g. Martin et al., 2020; C. Russell, 1990; Wei et al., 2010) to understand their formation mechanisms. At Earth, magnetic helicity was studied in magnetotail flux ropes (Zhang et al., 2010). A few FTE flux ropes were observed in the magnetotail flank with the positive sign of helicity under southward and duskward IMF conditions, indicating that they originated on the dayside and survived far downstream (Eastwood et al., 2012). Here we present a first dedicated study of the sign of helicity of FTEs at the Earth's dayside magnetopause.

Based on topological consideration, the helicity sign of FTEs should be controlled by the IMF. Figure 1 shows a schematic illustration of FTE formation by the multiple X-line reconnection mechanism under southward IMF with a non-zero $B_{Y}$ component. In 2-D (Figures 1a and 1c), as viewed from the dusk side, multiple reconnection between the magnetospheric and magnetosheath fields would produce a magnetic island (shown in purple) with an anti-clockwise field rotation. In 3-D, depending on the $B_{Y}$ (out-of-plane) component, the magnetic island becomes a magnetic flux rope with an axial component pointing outward (Figure 1a) or inward (Figure 1c) from the plane. The magnetic field rotation (tangential component) with respect to the axial direction of the flux rope determines its handedness or helicity sign. In this picture, the southward IMF with positive $B_{Y}$ would produce right-handed (RH) flux ropes (Figures 1a and $1 \mathrm{~b}$ ) while the southward IMF with negative $B_{Y}$ would produce left-handed (LH) flux ropes (Figures 1c and 1d). Such topological consideration has yet to be statistically tested.

We present a statistical study of FTEs observed by NASA's Magnetospheric Multiscale mission (MMS, Burch et al., 2015) and characterize the twist profiles of FTEs by fitting into a flux-rope model with systematic effort. We first introduce selections of FTEs, instrumentations, and illustration of events. We then present statistical analyses of the solar wind conditions. Finally, discussion and conclusions are presented.

\section{Data and Methods}

\subsection{Event Selections and Instrumentations}

We first obtain a list of FTEs as observed by MMS. Fargette et al. (2020) published a list of MMS1 observations of 229 FTEs consisting of 186 flux-rope-type structures and 43 flux-rope-type with reconnection at a central current sheet (e.g., interlinked flux tubes). An FTE is selected based on visual inspection of data 
(a)

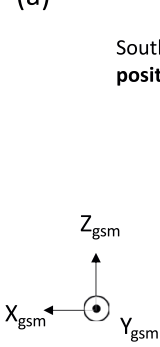

(c) Southward IMF + negative $B_{Y}$ (dawnward)

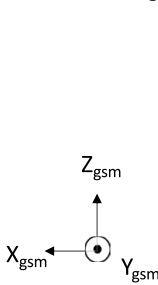

uthward IMF + ositive $B_{\mathrm{y}}$ (duskward)

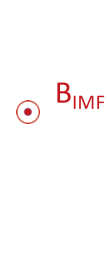

View from the Dusk

$\downarrow$

Right-handed flux rope

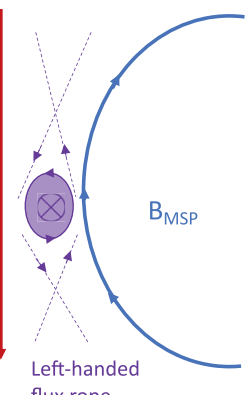

flux rope (b)

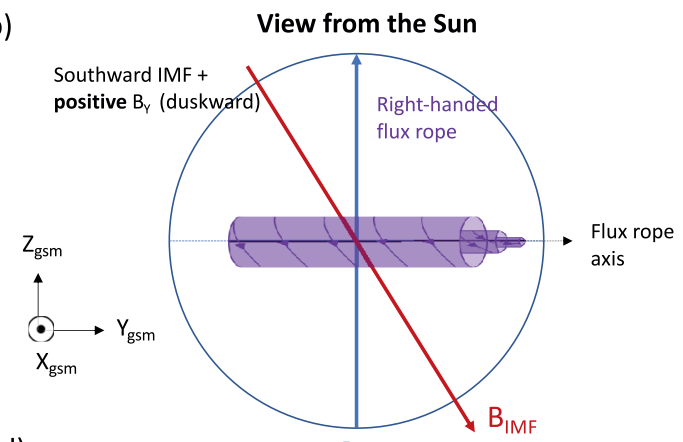

(d)

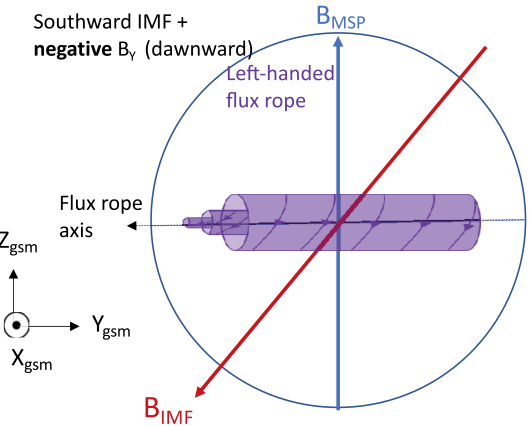

Figure 1. Schematic illustration of the generation of an FTE flux rope through the multiple X-line reconnection mechanism at the dayside magnetopause when the southward IMF has a significant (a), (b) positive $B_{Y}$ component and (c), (d) negative $B_{Y}$ component. The flux rope is shown in purple with arrows indicating the sense of twist when viewed from the dusk (a), (c) and the Sun (b), (d). When the flux rope is generated under southward IMF with positive $B_{Y}$, it has a right-handed sense of twist corresponding to a positive helicity. In contrast, when the flux rope is generated under southward IMF with negative $B_{Y}$, it has a left-handed sense of twist corresponding to a negative helicity. FTE, flux transfer event; IMF, interplanetary magnetic field.

plots in the Geocentric Solar Ecliptic (GSE) coordinates characterized by (1) a bipolar signature in one of the magnetic field components, and (2) an increase in the total (plasma and magnetic) pressure. We use Flux Gate Magnetometer (Russell et al., 2016) and Fast Plasma Instrument (Pollock et al., 2016) data in burst mode for the FTE intervals, and we only focus on events without reconnection (at a central current sheet) for our analyses (events with such sharp central current sheets typically do not fit a coherent flux rope structure). To analyze solar wind conditions preceding the FTEs, we obtain magnetic and velocity fields in GSE coordinates, IMF clock and cone angles in Geocentric Solar Magnetospheric (GSM) coordinates, plasma number density, dynamic pressure, Mach number, and plasma beta from the High-Resolution OMNI database (King \& Papitashvili, 2005). The IMF clock and cone angles are defined as $\arctan \left(B_{y} / B_{z}\right) \in\left[0^{\circ}, \pm 180^{\circ}\right]$ and $\arccos \left(B_{x} /|\mathbf{B}|\right) \in\left[0^{\circ}, 180^{\circ}\right]$, respectively.

\subsection{Flux Rope Fitting}

We perform a model fitting onto the data using a model first introduced by Burlaga (1988) to describe the magnetic flux rope structure of magnetic clouds in the solar wind (see also Lepping et al., 1990). The model assumes a cylindrically symmetric and force-free $(\nabla \times \mathbf{B}=\alpha \mathbf{B})$ configuration with a constant $\alpha$ in which the solution satisfying $\nabla^{2} B=-\alpha^{2} B$ was found by Lundquist (1950). The solution is in terms of the zeroth- and first-order Bessel functions; the axial component is modeled as $B_{A}=B_{0} J_{0}(\alpha R)$, the tangential (azimuthal) component as $B_{T}=B_{0} H J_{1}(\alpha R)$, and the radial component as $B_{R}=0$, where $H= \pm 1$ is the helicity sign, $B_{0}$ is the maximum field strength within the flux rope interval, and $R$ is the radial distance from the axis. From this model, we obtain a set of fit parameters $\left(\theta_{0}, \phi_{0}, Y_{0}, H\right)$ for each flux rope in the local observation frame $\left(\mathbf{x}_{\mathbf{v}}, \mathbf{y}_{\mathbf{v}}, \mathbf{z}_{\mathbf{v}}\right)$ coordinates (i.e., the flux rope's frame). To aid understanding, we reproduced an illustration from Burlaga (1988) in Figure S1. The $x_{v}$ is defined to be opposite to the flux rope motion direction such that $\mathbf{x}_{\mathbf{v}}=-\mathbf{V}_{\mathrm{AV}} /\left|\mathbf{V}_{\mathrm{AV}}\right|$, where $\mathbf{V}_{\mathrm{AV}}$ is the average velocity vector across the flux rope. The $\mathbf{z}_{\mathbf{v}}$ is calculated from $\pm \mathbf{X}_{\mathbf{v}} \times \mathbf{n}$, where $\boldsymbol{n}$ is the normal to the model magnetopause obtained from Shue et al. (1997), the positive (negative) sign is applied when the $Y$-component of $\mathbf{V}_{\mathrm{AV}}$ is positive (negative) to keep the $\mathbf{z}_{\mathbf{v}}$ pointing 

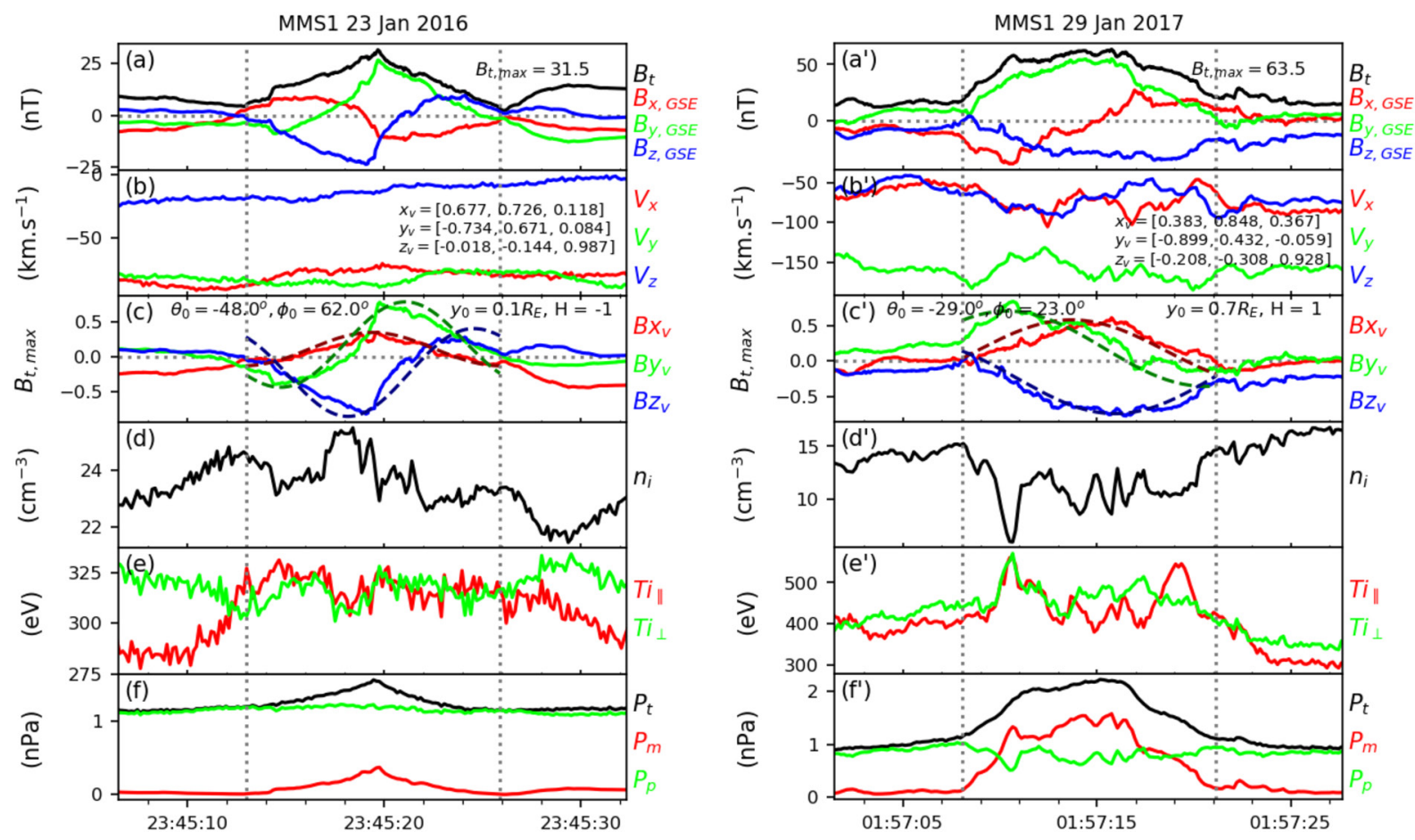

Figure 2. Overview of FTEs with (a)-(f) LH and (a'-f') RH flux rope structures. (a, a') Magnetic fields in GSE coordinates; (b, b') ion velocity in GSE coordinates with the transformations in text for the local frame coordinates; (c, $\left.c^{\prime}\right)$ magnetic fields in the local frame coordinates with the model parameters $\left(\theta_{0}\right.$, $\left.\phi_{0}, Y_{0}, H\right)$ and flux rope model; (d, d') ion number density; (e, e') ion temperature; and (f, $\left.\mathrm{f}^{\prime}\right)$ magnetic pressure, plasma pressure, and the total pressure. FTE, flux transfer event; GSE, Geocentric Solar Ecliptic; LH, left-handed; RH, right-handed.

northward (see Figure S1). Finally, $\mathbf{y}_{\mathbf{v}}=\mathbf{z}_{\mathbf{v}} \times \mathbf{x}_{\mathbf{v}}$ completes the orthonormal system. The angle $\theta_{0} \in\left[-90^{\circ}\right.$, $\left.90^{\circ}\right]$ is the angle of the flux rope axis from the ecliptic plane where $\theta_{0}=-90^{\circ}$ is southward and $\theta_{0}=90^{\circ}$ is northward. The angle $\phi_{0} \in\left[0, \pm 180^{\circ}\right]$ is the angle of the flux rope axis from the Sun-Earth line where the positive angle is duskward and negative angle is dawnward, and $Y_{0}$ is the impact parameter that is the $Y$ distance from the spacecraft to the flux rope model axis (see Figure S1), which is set to range from $-1 R_{E}$ to $1 R_{E}$. Note that the range of impact parameter is motivated by a typical cross-section of FTEs of $1 R_{E}$ (e.g., Sonnerup et al., 2004). The sense of helicity $H$ is +1 for $\mathrm{RH}$ or -1 for $\mathrm{LH}$ flux ropes. This handedness corresponds to the sense of rotation of the azimuthal (tangential) field around the flux rope axis: the rotation is anti-clockwise for RH flux ropes and it is clockwise for LH flux ropes when viewed from the direction where the axial field is pointing toward you. The four parameters are fitted onto the data by trial and error. An optimized set of parameters yield the minimum value of $\chi^{2}$ defined as $\chi^{2}=\sum_{i}^{N}\left(\left|\mathbf{B}_{\text {data }, i}-\mathbf{B}_{\text {model }, i}\right|^{2}\right) / N$ where $N$ is the number of vectors of magnetic field measurements. Examples of the model fitting results are shown in Figure 2.

Each flux rope is fitted for both helicity signs. The sign of helicity is then manually chosen based on visual inspection and comparison of the $\chi^{2}$ values of the two cases. Among the 186 flux ropes, we found that not all of them can be fitted well to the model, plausibly due to the fact that those flux ropes are not totally forcefree. Also, since we will investigate the solar wind conditions preceding these events, we exclude events for which OMNI data are missing. We select 84 flux ropes that are well fitted to the model based on visual inspection (i.e., low $\chi^{2}$ value). Note that all events are in the northern winter hemisphere (September-February) due to the MMS orbit that samples data near the subsolar region during this time of year. Table S1 lists the time intervals of these flux ropes along with their fit parameters $\left(\theta_{0}, \phi_{0}, Y_{0}, H\right)$ and $\chi^{2}$. 

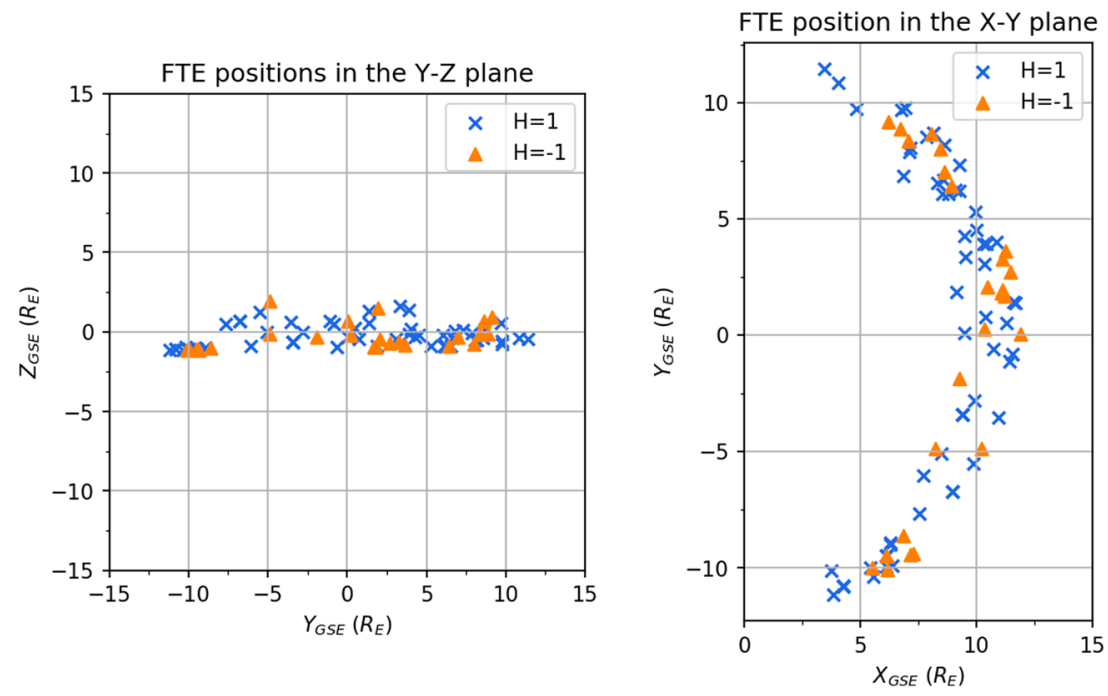

Figure 3. Spatial distribution of the FTE flux ropes in the (a) Y-Z and (b) X-Y planes with data points color-coded by the helicity signs $H=1(\mathrm{RH})$ represented by blue cross and $H=-1(\mathrm{LH})$ represented by orange triangle. Both types of flux rope uniformly distribute on the dayside magnetopause. FTE, flux transfer event; LH, left-handed; RH, right-handed.

\section{Event Illustrations and Statistical Analyses}

\subsection{Event Illustrations}

Figure 2 shows examples of LH (left) and RH (right) flux ropes, observed by MMS1 on January 23, 2016 at 23:45 UT and January 29, 2017 at 1:57 UT, respectively. MMS1 was located at $[7.3,-9.4,-1.1]_{\mathrm{GSE}} R_{E}$ for the first event and $[9.9,-5.5,1.2]_{\mathrm{GSE}} R_{E}$ for the second event. The average IMF clock angles during an interval of $15 \mathrm{~min}$ before the first and second events are $-162^{\circ} \pm 3$ and $114^{\circ} \pm 4$, respectively (i.e., southward). The bipolar magnetic variations are seen in the $B_{X}$ component (as expected for a magnetopause normal orientation near the sub-solar point) while the enhanced core field is seen in $B_{Y}$ component for both events (Figures 1a and $\left.1 \mathrm{a}^{\prime}\right)$ in the GSE coordinates. However, the senses of rotation of $B_{X}$ are opposite in each case. To move to the flux rope's frame, we obtain the $\left(\mathbf{x}_{\mathbf{v}}, \mathbf{y}_{\mathbf{v}}, \mathbf{z}_{\mathbf{v}}\right)$ coordinates as described in Section 2.2 (see also Figure S1) from the average ion bulk velocity during the intervals in Figures $2 b$ and $2 b^{\prime}$, bounded by the vertical dotted lines. The magnetic fields are then transformed to this local observation frame and normalized with the maximum magnetic field strength, called $\left(B_{\mathrm{xv}}, B_{\mathrm{yv}}, B_{\mathrm{zv}}\right)$, in Figures $1 \mathrm{c}$ and $1 \mathrm{c}^{\prime}$ for the purpose of fitting into the model. The fitting results to the Burlaga model are plotted as dashed lines in Figures $1 \mathrm{c}$ and $1 \mathrm{c}^{\prime}$, along with the fit parameters in text in the same panels. The model fitting in Figure $2 \mathrm{c}$ shows that it has a negative helicity (LH) while in Figure $1 \mathrm{c}^{\prime}$ it has a positive helicity (RH); the opposite sense of twist is seen in $B_{\mathrm{yv}}$ component. Figures $2 \mathrm{~d}\left(2 \mathrm{~d}^{\prime}\right), 2 \mathrm{e}\left(2 \mathrm{e}^{\prime}\right)$, and $2 \mathrm{f}\left(2 \mathrm{f}^{\prime}\right)$ show variations in ion number density, ion temperature, and plasma, magnetic, and total pressure across the two flux rope intervals, respectively.

\subsection{Spatial Distribution of FTEs}

Among the 84 FTE flux ropes, we found that there are 59 (70\%) RH flux ropes and 25 (30\%) LH flux ropes. Figure 3 shows the spatial distribution of FTE locations in the Y-Z and X-Y planes in the GSE coordinates for the RH (blue cross) and LH (orange triangle) flux ropes. As seen in Figure 3, the positive (RH) and negative (LH) helicity flux ropes uniformly distribute on the dayside magnetopause with their positions being at low-latitudes. In other words, there is no spatial preference for FTE flux rope's handedness. This suggests that the sense of twist is not related to these local properties but should be associated with remote or upstream parameters.

It is important to note that the handedness is different from the sequence of polarity of the bipolar variation of FTEs that is observed dependent on the hemisphere (e.g., Rijnbeek et al., 1984; Southwood et al., 1986). The bipolar variation is observed to be outward followed by inward to the magnetopause for spacecraft 
(a)

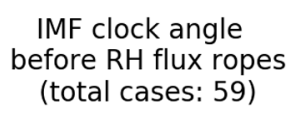

IMF clock angle

before RH flux ropes

(total cases: 59)

$0^{\circ}$

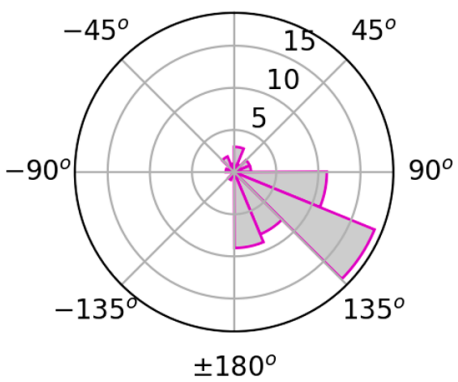

(c)

$$
\begin{gathered}
\text { before regular LH flux ropes } \\
\text { (total cases: 16) }
\end{gathered}
$$

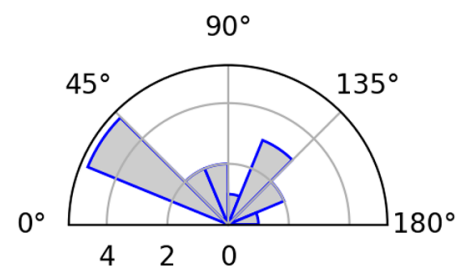

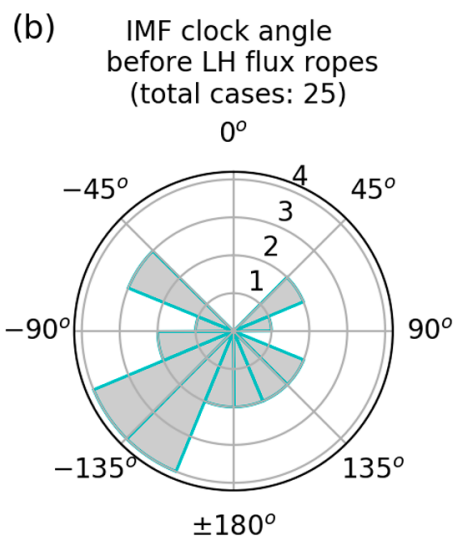

(d) IMF cone angle before outlier LH flux ropes (total cases: 9)

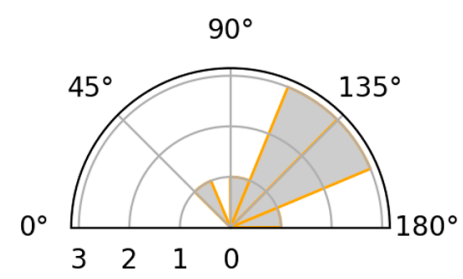

Figure 4. (top) Distribution of the averaged IMF clock angle during an interval of 15 minutes before the FTE observations obtained from the OMNI database for (a) RH and (b) LH flux ropes. The IMF clock angle is mainly in the $90^{\circ}$ to $180^{\circ}$ clock angle range (duskward-southward) before the RH flux ropes and in the $-90^{\circ}$ to $-180^{\circ}$ range (dawnward-southward) before the LH flux ropes. (bottom) Distribution of the averaged IMF cone angle during an interval of 15 minutes before LH flux ropes for (c) regular group and (d) outlier group. The regular LH flux ropes are mostly preceded by sunward-tilted IMF $B_{x}$ while the outlier LH flux ropes are mainly preceded by antisunward-tilted IMF $B_{x}$. FTE, flux transfer event; GSE, Geocentric Solar Ecliptic; IMF, interplanetary magnetic field; LH, left-handed; $\mathrm{RH}$, right-handed.

located in the northern hemisphere; this order is reversed for spacecraft located in the southern hemisphere. Both sequences can have the same helicity sign as the order of sequence depends on the relative spacecraft trajectory; the direction of the core field with respect to the bipolar variation is what determines the helicity sign of the flux rope.

\subsection{Solar Wind IMF Conditions}

We studied solar wind conditions using their averages during an interval of 15 minutes before the events, for all FTE flux ropes. We also analyzed the average solar wind conditions during intervals of 5, 10, 20, and 25 minutes; the qualitative results remained unchanged. Figures $4 \mathrm{a}$ and $4 \mathrm{~b}$ show histograms of the averaged IMF clock angle during an interval of 15 minutes before the two types of events (RH and LH). It is found that the RH flux ropes are mainly preceded by IMF clock angles from $90^{\circ}$ to $180^{\circ}$, which is the duskward and southward direction. In contrast, the LH flux ropes are mainly preceded by IMF clock angles of $-90^{\circ}$ to $-180^{\circ}$, which is dawnward and southward. We do not find any clear correlation between other solar wind parameters and the helicity sign of flux ropes (see Figure S2). This suggests that the helicity sign of FTE flux ropes is mainly controlled by the IMF clock angle (e.g., the IMF $B_{Y}$ component). Nevertheless, there are clearly some LH flux ropes ( 9 out of 25 cases) that are preceded by duskward IMF (IMF $B_{Y}>0$ ) and some $\mathrm{RH}$ flux ropes ( 5 out of 59 cases) that are preceded by dawnward IMF (IMF $B_{Y}<0$ ), henceforth referred to as outliers. The smaller outlier population for the RH flux ropes may relate to the seasonal (i.e., the dipole tilt) effects because all events are observed during September-February which are near the winter solstice (December). Note that not all of the outlier events are preceded by southward IMF; a significant population of the outlier RH (4 out of 5) and LH ( 3 out of 9) flux ropes are preceded by northward IMF. Excluding the 
northward IMF events, it is still unclear whether this outlier group is due to statistical uncertainties, such as IMF propagation errors, or to some unknown physical mechanism controlling the helicity in addition to the IMF $B_{Y}$.

For the purpose of discussion and further analyses, we define two groups of LH flux ropes to be (1) "regular" for LH flux ropes that are preceded by dawnward IMF and (2) "outlier" for LH flux ropes that are preceded by duskward IMF. Note that we study only the LH flux group due to the significant outlier population. Comparing the solar wind conditions between the two groups, we found that they have different IMF cone angle as shown in Figures $4 \mathrm{c}$ and $4 \mathrm{~d}$. The regular group is mostly preceded by IMF cone angle $<90^{\circ}$ (sunward) while the outlier group is mainly preceded by IMF cone angle $>90^{\circ}$ (anti-sunward). The magnitudes of IMF $B_{x} /|\mathbf{B}|$ are also different (see panel (c) of Figure S3). The outlier group has mostly negative IMF $B_{x} /|\mathbf{B}|$ values and large magnitude. However, the regular group has a weak, positive IMF $B_{x} /|\mathbf{B}|$. There is no significant difference in other solar wind parameters between these groups.

\section{Discussion}

We have analyzed the helicity sign of 84 FTE flux ropes observed by MMS1 (from a list by Fargette et al., 2020) at the dayside magnetopause through model fitting. We found that there are $59 \mathrm{RH}(70 \%)$ and $25(30 \%)$ LH flux ropes. We also analyzed the solar wind conditions preceding the events. We found a correlation between the IMF $B_{Y}$ sign and the helicity sign: RH flux ropes $(H=1)$ are mainly preceded by IMF $B_{Y}>0$ while LH flux ropes $(H=-1)$ are mostly preceded by IMF $B_{Y}<0$. This shows that the twist direction of the FTE flux ropes is controlled by the IMF $B_{Y}$ component.

Our main results place constraints on the FTE generation mechanism. Indeed, as we illustrate in Figure 1, the helicity sign of an FTE can be predicted as a function of the IMF $B_{Y}$ in the context of a multiple, sequential X-line formation mechanism. The sense of rotation of the azimuthal field of the flux ropes from our statistical analyses can be explained by this picture where the FTEs are generated by multiple component reconnection X-lines on the dayside magnetopause as predicted by Lee and Fu (1985). Additionally, most events are observed near the winter solstice, that is, when the dipole tilt is large, consistent with the FTE production due to sequential, multiple X-line mechanism proposed by Raeder (2006). As in the Raeder's model, with a positive dipole tilt (toward the Sun), the stagnation point of the flow shifts northward away from the subsolar point. Consequently, a reconnection X-line is formed one after the other while an FTE is generated between these sequential reconnection X-lines. This is not the same as the bursty single X-line model (Scholer, 1988; Southwood et al., 1988) where an FTE is generated as a consequence of the unsteady reconnection rate. Indeed, the Maximum Magnetic Shear Model (Trattner et al., 2007) predicted that the component reconnection should be dominant on the dayside when the southward IMF has a significant $B_{Y}$ component because the draped IMF in the magnetosheath region makes a first contact with the subsolar region. The FTEs should be generated from this region through multiple X-line reconnection where the sign of IMF $B_{Y}$ across the neutral line controls the sense of twist and core field as depicted in Figure 1. Even though our finding does not rule out other FTE formation mechanisms, it is consistent and compatible with the multiple X-line mechanism.

Statistical analyses on the solar wind conditions prior to the LH flux ropes preceded by IMF $B_{Y}>0$ (outlier) and those preceded by IMF $B_{Y}<0$ (regular) show that the outlier group has a strong, negative IMF $B_{X}$ while the regular group has a small, positive IMF $B_{X}$. It is unclear whether the magnitude and polarity of IMF $B_{X}$ should control the helicity sign of FTE flux ropes. When the IMF is due south and $B_{X}$ is negative, the magnetic merging line is found to shift southward at the dayside (Peng et al., 2010). In addition, with all our events being in the winter hemisphere, there is plausibly a combined effect between the IMF $B_{X}$ and the dipole tilt (e.g., Hoilijoki et al., 2014; Palmroth et al., 2012) that can complicate reconnection at the dayside and thus the FTE formation. The IMF $B_{X}$ component was found to impact the north-south hemispheric asymmetry of FTE occurrence, properties, sizes, and motions (Hoilijoki et al., 2019) as a result of a reduction of the reconnection rate at the dayside due to the smaller tangential magnetic field to the magnetopause. The FTE generation may also be complicated by processes downstream of bow shock when the IMF cone angle is small. We leave this as an open question that should be addressed in future work. 


\section{Conclusions}

We have surveyed the helicity sign of 84 FTE flux ropes observed by MMS near the winter solstice during years 2015-2017 that can be fitted well to a cylindrically force-free flux rope model with a constant $\alpha$ (Burlaga, 1988). We found that 59 (70\%) flux ropes are $\mathrm{RH}$ and $25(30 \%)$ of them are LH. Investigations of the IMF conditions show that the RH flux ropes are mainly preceded by southward IMF with positive $B_{Y}$ while the LH flux ropes are mostly preceded by southward IMF with negative $B_{Y}$. This control of FTE flux rope helicity sign by the IMF $B_{Y}$ component is consistent with its formation through sequential, multiple X-line reconnection. We also found an outlier group of flux ropes whose helicity sign is opposite to that of IMF $B_{y}$, which is unexpected. There are 14 out of 84 flux ropes that are preceded by unexpected IMF $B_{Y}$ polarity. Investigation of the solar wind conditions preceding LH flux ropes show that the outlier group is associated with strong and negative IMF $B_{X}$. This shows that the presence of IMF $B_{X}$ further complicates the formation of FTE flux ropes at the dayside magnetopause. Future work would be desirable for a fuller understanding of FTE helicity generation of this outlier group.

\section{Data Availability Statement}

Data are handled using SpacePy (Morley et al., 2011) and Pandas (McKinney, 2010) packages and plotted using Matplotlib (Hunter, 2007) and Seaborn (Waskom et al., 2017) packages with Python 3.

\section{References}

Work at IRAP was supported by CNRS, CNES, and UPS. MMS data are available online (from https://lasp. colorado.edu/mms/sdc/public/). The upstream solar wind conditions are obtained from the OMNIweb database (accessible from https://omniweb.gsfc. nasa.gov/). We thank Emmanuel Penou for developing the CL software that was used to visualize and obtain the data (available at https://clweb.irap.omp.eu/ cl/clweb.php).
Berchem, J., \& Russell, C. T. (1984). Flux transfer events on the magnetopause: Spatial distribution and controlling factors. Journal of Geophysical Research, 89(A8), 6689. https://doi.wiley.com/10.1029/JA089iA08p06689

Bothmer, V., \& Schwenn, R. (1998). The structure and origin of magnetic clouds in the solar wind. Annales Geophysicae, 16(1), 1-24. https://doi.org/10.1007/s00585-997-0001-X

Burch, J., Moore, T. E., Torbert, R., \& Giles, B. L. (2015). Magnetospheric multiscale overview and science objectives. Space Science Reviews, 199, 5-21. https://doi.org/10.1007/s11214-015-0164-9

Burlaga, L. F. (1988). Magnetic clouds and force-free fields with constant alpha. Journal of Geophysical Research, 93(A7), 7217. https://doi. org/10.1029/JA093iA07p07217

Cowley, S. W. H. (1982). The causes of convection in the Earth's magnetosphere: A review of developments during the IMS. Reviews of Geophysics, 20(3), 531. https://doi.org/10.1029/RG020i003p00531

Eastwood, J. P., Phan, T. D., Fear, R. C., Sibeck, D. G., Angelopoulos, V., Øieroset, M., \& Shay, M. A. (2012). Survival of flux transfer event (FTE) flux ropes far along the tail magnetopause. Journal of Geophysical Research, 117(A8). https://doi.org/10.1029/2012JA017722

Fargette, N., Lavraud, B., Øieroset, M., Phan, T. D., Toledo-Redondo, S., Kieokaew, R., et al. (2020). On the ubiquity of magnetic reconnection inside flux transfer event-like structures at the Earth's magnetopause. Geophysical Research Letters, 47(6), 1-9. https://doi. org/10.1029/2019GL086726

Fear, R. C., Milan, S. E., \& Oksavik, K. (2012). Determining the axial direction of high-shear flux transfer events: Implications for models of FTE structure. Journal of Geophysical Research: Space Physics, 117(9), 1-19. https://doi.org/10.1029/2012JA017831

Hasegawa, H., Wang, J., Dunlop, M., Pu, Z., Zhang, Q. H., Lavraud, B., et al. (2010). Evidence for a flux transfer event generated by multiple X-line reconnection at the magnetopause. Geophysical Research Letters, 37, L16101. https://doi.org/10.1029/2010GL044219

Hoilijoki, S., Ganse, U., Sibeck, D. G., Cassak, P. A., Turc, L., Battarbee, M., et al. (2019). Properties of magnetic reconnection and FTEs on the dayside magnetopause with and without positive IMF B x component during southward IMF. Journal of Geophysical Research: Space Physics, 124(6), 4037-4048. https://doi.org/10.1029/2019JA026821

Hoilijoki, S., Souza, V. M., Walsh, B. M., Janhunen, P., \& Palmroth, M. (2014). Magnetopause reconnection and energy conversion as influenced by the dipole tilt and the IMF Bx. Journal of Geophysical Research: Space Physics, 119(6), 4484-4494. https://doi. org/10.1002/2013JA019693

Hunter, J. D. (2007). Matplotlib: A 2d graphics environment. Computing in Science \& Engineering, 9(3), 90-95.

Karlson, K. A., Øieroset, M., Moen, J., \& Sandholt, P. E. (1996). A statistical study of flux transfer event signatures in the dayside aurora: The IMF B y -related prenoon-postnoon symmetry. Journal of Geophysical Research, 101(A1), 59-68. https://doi.org/10.1029/95JA02590

King, J. H., \& Papitashvili, N. E. (2005). Solar wind spatial scales in and comparisons of hourly wind and ace plasma and magnetic field data. Journal of Geophysical Research, 110(A2). https://doi.org/10.1029/2004JA010649

Kuo, H., Russell, C. T., \& Le, G. (1995). Statistical studies of flux transfer events. Journal of Geophysical Research, 100(A3), 3513-3519. https://doi.org/10.1029/94JA02498

Lee, L., \& Fu, Z. (1985). A theory of magnetic flux transfer at the Earth's magnetopause. Geophysical Research Letters, 12, 105-108. https:// doi.org/10.1029/GL012i002p00105

Lepping, R. P., Jones, J. A., \& Burlaga, L. F. (1990). Magnetic field structure of interplanetary magnetic clouds at 1 AU. Journal of Geophysical Research, 95(A8), 11957. https://doi.org/10.1029/ja095ia08p11957

Lundquist, S. (1950). Magnetohydrostatic fields. Ark Fys, 2, 361-365.

Martin, C. J., Arridge, C. S., Badman, S. V., Russell, C. T., \& Wei, H. (2020). Distribution and properties of magnetic flux ropes in Titan's ionosphere. Journal of Geophysical Research: Space Physics, 125(4), 1-14. https://doi.org/10.1029/2019JA027570

McKinney, W. (2010). Data structures for statistical computing in python. In S. van der Walt, \& J. Millman (Eds.), Proceedings of the 9th python in science conference (pp. 51-56), Zenodo.

Morley, S. K., Koller, J., Welling, D. T., Larsen, B. A., Henderson, M. G., \& Niehof, J. T. (2011). Spacepy-A Python-based library of tools for the space sciences. In Proceedings of the 9th Python in science conference (SciPy 2010). Austin, TX: Zenodo. 
Øieroset, M., Phan, T. D., Eastwood, J. P., Fujimoto, M., Daughton, W., Shay, M. A., et al. (2011). Direct evidence for a three-dimensional magnetic flux rope flanked by two active magnetic reconnection $x$ lines at earth's magnetopause. Physical Review Letters, $107,165007$. https://doi.org/10.1103/PhysRevLett.107.165007

Palmroth, M., Fear, R. C., \& Honkonen, I. (2012). Magnetopause energy transfer dependence on the interplanetary magnetic field and the Earth's magnetic dipole axis orientation. Annales Geophysicae, 30(3), 515-526. https://doi.org/10.5194/angeo-30-515-2012

Paschmann, G., Haerendel, G., Papamastorakis, I., Sckopke, N., Bame, S. J., Gosling, J. T., \& Russell, C. T. (1982). Plasma and magnetic field characteristics of magnetic flux transfer events. Journal of Geophysical Research, 87(A4), 2159-2168. https://doi.org/10.1029/ JA087iA04p02159

Peng, Z., Wang, C., \& Hu, Y. Q. (2010). Role of IMF Bx in the solar wind-magnetosphere-ionosphere coupling. Journal of Geophysical Research, 115(8), 1-7. https://doi.org/10.1029/2010JA015454

Pollock, C., Moore, T., Jacques, A., Burch, J., Gliese, U., Saito, Y., et al. (2016). Fast plasma investigation for magnetospheric multiscale. Space Science Reviews, 199, 331-406. https://doi.org/10.1007/s11214-016-0245-4

Raeder, J. (2006). Flux transfer events: 1. Generation mechanism for strong southward IMF. Annales Geophysicae, 24(1), 381-392. https:// doi.org/10.5194/angeo-24-381-2006

Rijnbeek, R. P., Cowley, S. W. H., Southwood, D. J., \& Russell, C. T. (1984). A survey of dayside flux transfer events observed by ISEE 1 and 2 magnetometers. Journal of Geophysical Research, 89(A2), 786. https://doi.org/10.1029/JA089iA02p00786

Russell, C. (1990). Magnetic flux ropes in the ionosphere of Venus. Washington DC American Geophysical Union Geophysical Monograph Series, 58, 413-423. https://doi.org/10.1029/GM058p0413

Russell, C., \& Elphic, R. (1978). Initial ISEE magnetometer results: Magnetopause observations. Space Science Reviews, $22,681-715$.

Russell, C., Le, G., \& Kuo, H. (1996). The occurrence rate of flux transfer events. Advances in Space Research, 18(8), 197-205. https://doi. org/10.1016/0273-1177(95)00965-5

Russell, C. T., Anderson, B. J., Baumjohann, W., Bromund, K. R., Dearborn, D., Fischer, D., et al. (2016). The magnetospheric multiscale magnetometers. Space Science Reviews, 199(1-4), 189-256. https://doi.org/10.1007/s11214-014-0057-3

Saunders, M. A., Russell, C. T., \& Sckopke, N. (1984). Flux transfer events: Scale size and interior structure. Geophysical Research Letters, 11(2), 131-134. https://doi.org/10.1029/GL011i002p00131

Scholer, M. (1988). Magnetic flux transfer at the magnetopause based on single X line bursty reconnection. Geophysical Research Letters 15(4), 291-294. https://doi.org/10.1029/GL015i004p00291

Shue, J. H., Chao, J. K., Fu, H. C., Russell, C. T., Song, P., Khurana, K. K., et al. (1997). A new functional form to study the solar wind control of the magnetopause size and shape. Journal of Geophysical Research, 102(A5), 9497-9512. https://doi.org/10.1029/97JA00196

Song, Y., \& Lysak, R. L. (1989). Evaluation of twist helicity of flux transfer event flux tubes. Journal of Geophysical Research, 94(A5), 5273. https://doi.org/10.1029/JA094iA05p05273

Sonnerup, B. U. Ö., Hasegawa, H., \& Paschmann, G. (2004). Anatomy of a flux transfer event seen by Cluster. Geophysical Research Letters, 31(11), L11803. https://doi.org/10.1029/2004GL020134

Southwood, D., Saunders, M., Dunlop, M., Mier-Jedrzejowicz, W., \& Rijnbeek, R. (1986). A survey of flux transfer events recorded by the UKS spacecraft magnetometer. Planetary and Space Science, 34(12), 1349-1359. https://doi.org/10.1016/0032-0633(86)90071-1

Southwood, D. J., Farrugia, C. J., \& Saunders, M. A. (1988). What are flux transfer events? Planetary and Space Science, 36(5), 503-508. https://doi.org/10.1016/0032-0633(88)90109-2

Trattner, K. J., Mulcock, J. S., Petrinec, S. M., \& Fuselier, S. A. (2007). Probing the boundary between antiparallel and component reconnection during southward interplanetary magnetic field conditions. Journal of Geophysical Research, 112(8), 1-16. https://doi. org/10.1029/2007JA012270

Wang, Y. L., Elphic, R. C., Lavraud, B., Taylor, M. G., Birn, J., Russell, C. T., et al. (2006). Dependence of flux transfer events on solar wind conditions from 3 years of Cluster observations. Journal of Geophysical Research, 111(4), 1-13. https://doi.org/10.1029/2005JA011342

Waskom, M., Botvinnik, O., O'Kane, D., Hobson, P., Lukauskas, S., Gemperline, D. C., et al. (2017). Mwaskom/seaborn: v0.8.1. Zenodo. https://doi.org/10.5281/zenodo.883859

Wei, H. Y., Russell, C. T., Zhang, T. L., \& Dougherty, M. K. (2010). Comparison study of magnetic flux ropes in the ionospheres of Venus, Mars and Titan. Icarus, 206(1), 174-181. https://doi.org/10.1016/j.icarus.2009.03.014

Wright, A. N., \& Berger, M. A. (1989). The effect of reconnection upon the linkage and interior structure of magnetic flux tubes. Journal of Geophysical Research, 94(A2), 1295. https://doi.org/10.1029/JA094iA02p01295

Wright, A. N., \& Berger, M. A. (1990). The interior structure of reconnected flux tubes in a sheared plasma flow. Journal of Geophysical Research, 95(A6), 8029. https://doi.org/10.1029/JA095iA06p08029

Zhang, Y. C., Shen, C., Liu, Z. X., \& Narita, Y. (2010). Magnetic helicity of a flux rope in the magnetotail: THEMIS results. Annales Geophysicae, 28(9), 1687-1693. https://doi.org/10.5194/angeo-28-1687-2010 